\title{
Structural Identification of Systems with Distributed Lag
}

\author{
Nikolay Karabutov, Dr., Prof. \\ Dept. of Problems Control, Moscow State Engineering University of Radio Engineering, Electronics and Automation, \\ Dept. of Mathematics, Financial University under the Government of the Russian Federation, Moscow, Russia \\ E-mail:nik.karabutov@gmail.com;kn22@yandex.ru
}

\begin{abstract}
The problem of structural identification of systems with the distributed lag in the conditions of uncertainty is considered. Known statistical approaches are laborious and not always allow making the decision on lag structure. Therefore in work for the problem decision the special class of static structures (SS) (virtual portraits) explored system is introduced. Process of the decision of a problem consists of two steps. At the first step set of secants for initial system is under construction. Completeness of set of secants is a sign of linearity of system. Nonfulfilment of conditions of completeness is a sign of nonlinearity of system. Estimation of nonlinearity of system execute on an indicator of level of nonlinearity of the system, offered in work. At the second step the special structural space is introduced and is defined SS for a nonlinear part of system. The estimation of nonlinear properties of system is executed on the basis of identification of parameters of set of secants SS. Criteria and algorithms of decision-making on structure of a lag on the bas is of the analysis of virtual portraits are offered. The analogue of criterion of Durbin-Watson is offered. The received results are generalized on a case of the distributed lag in input and output variables of system. It is shown that to structural identification of systems with the distributed lag we will not apply the analysis of sector sets. The approach to parametrical identification of system with the distributed lag in the conditions of uncertainty is offered.
\end{abstract}

Index Terms - Structural Identification, Distributed Lag, Virtual Portrait, Decision-Making, Parametrical Identification

\section{Introduction}

Models with the distributed lags are widely applied in an econometrics and economy [1-4], the engineering [57] and medicine [8-10]. Delay can have both independent, and dependent variables. The account of the distributed lag activates autocorrelation between variables $[1,2,4]$ and complicates process of identification parameters object. To identification of parameters system apply various models of approximation parameters at the distributed lags. Such approach allows reducing number of estimated parameters of system. The Koyck scheme [2, 12], based on a change of factors of model on a decreasing geometrical progression is most widely applied. The Fisher model $[1,11]$ is based on a change of factors model on the set decreasing arithmetical progression. Fisher scheme are applied in that case when object parameters decrease from the first me mbers of a progression. S. Almon [13] modified Fisher model, having applied the polynomial law of change factors. Other approaches to the definition of laws change parameters at the distributed lags are described in [4, 11, 14, 15]. The accounting of a priori information on dependence between the distributed lags in the form of some rational polynom is stated in [16].

The considered models of factors minimize number of unknown parameters. To an estimation of parameters apply a method least-squares method [1-4]. In these works the model structure is postulated a priori and the problem of parametrical identification is considered. In [17] the interactive algorithm of an estimation parameters static plant with the distributed lags is offered. The length of a lag is set and does not become any assumptions of interdependence between system parameters. The case of a piecewise monotonic change of parameters plant is considered. In a number of works methods of a choice maximum length a lag are offered. They are founded on application the statistics, the rests based on the analysis $[1,15,18]$. In $[19,20]$ various methods of an estimation parameters model with the distributed lag in a case of a priori set structure of model are considered. In [21] influence of a priori information, received from the analysis of the empirical data, on a choice of structure model with the distributed lag on an output variable is researched. Polynomial schemes for estimation parameters are offered. In work [22] are described algorithm of an estimation parameters model with the distributed lag. On the basis of results modelling the structure of model which explains a mis match of model applied now in the American interest rate on federal funds is selected. In [23] process of inflation with the help autoregressive models is researched. The choice of length a lag is carried out on the basis of consecutive magnification delays and an estimation of adequacy the received model. The choice of length a lag is based on step-by-step increase in delays and an estimation of adequacy the received model. Then the Akaike criterion 
and Bayesian information criteria are applied and the solution is made on model structure. The case of a priori uncertainty concerning structure and plant parameters was not study.

In work [24] the review of existing approaches to construction of models of an econometrics is executed. The analysis is based on methodology general-tospecific. It is based on simplification of the general theoretical model for the purpose of its conformity to experimental data. The methodology is based on statistical modelling and application of known statistics for a choice of length a lag. Authors consider application of the given approach to construction of models both with the dynamic specification of lag, and with a lag in an independent variable. The combination of methods of modelling to application of various statistics is most widely applied to a choice of structure the distributed lag [for example, 25, 26, 27]. In the majority of works disadvantages of existing statistics are considered and the area of their application is underlined.

In work [28] the information-set approach to structural identification of systems with the distributed lag in case of an irregular input [29] is offered. It is based on the analysis of static structures (visual portraits), reflecting nonlinear properties of system in special structural space [29]. Algorith ms of decision-making on the maximum length of the distributed lag are offered. Algorithms do not demand calculation of statistical criteria. The analogue of Durbin-Watson criterion for a considered case is offered.

In the given work the approach offered in [28] develops. Criteria of decision-making on length of the distributed lag on input and output variables of system are described. It is shown, that for structural identification of systems with the distributed lag sector sets $[28,30]$ are not applicable. The approach to parametrical identification of system in the conditions of uncertainty is offered.

Article has the following structure. At first the method of an estimation of degree of linearity of system is described. The following section contains the description of a method of reception of set of the data for an estimation of structure of the distributed lag. In the subsequent sections the received results are applied to a choice of length of a lag on the basis of input and output variables. It is shown that sector sets are inapplicable for a choice of structure of a lag. In final section the problem of identification parameters of system with the distributed lag is analyzed. Results of modelling are resulted.

\section{Problem Statement}

Consider plant

$$
y_{n}=A_{n}^{T} U_{n}+\xi_{n}
$$

where $y_{n} \in R$ is an exit; $U_{n} \in R^{k}$ is the input vector which elements are limited, is limiting nondegenerate functions; $n \in J_{N}=[0, N]$ is discrete time, $N<\infty$; $X_{n}=X\left(u_{i, n} \in U_{n}, y_{n}\right) \in R^{n}$ is a vector of the distributed lags on $u_{i, n} \in U_{n}$ and $y_{n} ; A \in R^{k}, B \in R^{m}$ are vectors of constant parameters; $\xi_{n} \in R$ is a perturbation, $\left|\xi_{n}\right|<\infty$ for all $n \in J_{N}$.

We believe, as $u_{i, n} \in U_{n}(i=\overline{1, k})$ and $\xi_{n} \in R$ are irregular functions of time.

For (1) the set of the measured values is known

$$
\mathrm{I}_{o}=\left\{y_{n}, U_{n} \forall n \in J_{N}\right\}
$$

and map $\Gamma_{o}:\left\{U_{n}\right\} \times\left\{y_{n}\right\} \quad \forall n \in J_{N}$ describing an observable informational portrait [7, 29].

It is necessary $\mathrm{I}_{o}, \Gamma_{o}$ to estimate on the basis of analysis structure of system (1). It means that it is necessary to estimate degree of linearity and dimension of vector $X_{n}$.

\section{Estimation of Degree of Linearity System (1)}

Consider restriction of an observable information portrait $\left.\Gamma_{o}^{u_{i}} \subset \Gamma_{o}\right|_{u_{i} \in U} \forall i=\overline{1, k}$ and for every $\Gamma_{o}^{u_{i}}$ construct a secant

$$
\bar{\gamma}\left(y, u_{i}\right)=a_{i} u_{i, n},
$$

where $a_{i}$ are some real numbers.

Introduce set on (2) set of secants for $\Gamma_{o}$

$$
\mathrm{S}(U, y)=\left\{\bar{\gamma}\left(y, u_{i}\right) \quad \forall i=\overline{1, k}\right\}
$$

Definition 1 [7, 29]. Field of structures $S_{S}$ of system(1) name set of maps $\bar{\gamma}\left(y, u_{i}\right):\left\{u_{i}\right\} \rightarrow\{y\} \quad \forall i=\overline{1, k}$ on Euclidean plane $\mathrm{E}$

$$
S_{S}=\mathrm{S}(U, y) .
$$

Designate

$$
\Phi_{n}=\left[\bar{\gamma}\left(y_{n}, u_{1, n}\right), \bar{\gamma}\left(y_{n}, u_{2, n}\right), \ldots, \bar{\gamma}\left(y_{n}, u_{k, n}\right)\right]^{T}
$$

and consider the equation

$$
\hat{y}_{n}=\Psi^{T} \Phi_{n},
$$

where vector $\Psi \in R^{k}$ define by means of a leastsquares method. Estimation $\Psi$ exists on the basis of the suppositions made in section 2 concerning input $U_{n}$. 
Completeness of system (1) in the field of structures $S_{S}$ follows from the following statement [29, 31].

Theorem 1. Consider a vector of in formative variables $U_{n} \in R^{k}$ and a field of structures $S_{S} \subset \mathrm{S}(U, y)$ for (1). Then the field of structures $S_{S}$ of system(1) is full, if

$$
\sum_{i=1}^{k} \psi_{i}=1
$$

where $\psi_{i}$ is $i$-th element of vector $\Psi$ in (4).

The theorem 1 gives sufficient conditions of linearity (nonlinearity, collinearity) systems (1) on the set field of structures. If the condition (4) is fulfilled, that field $S_{S}$ is full. Hence, $S_{S}$ is a linear span of an exit system (1). Otherwise make a solution on presence of nonlinearity or collinearity (autocorrelation) in system(1).

Let

$$
\sum_{i=1}^{k} \psi_{i}=\chi
$$

Magnitude $\Delta(\Psi)=\chi-1$ name level of nonlinearity of system (1) in parametrical space $\mathscr{P}_{\mathbb{P}}=(A, B)$. As the distributed lags are the multicollinearity reason in (1), then $\Delta(\Psi)$ will accept small values.

\section{Set for an Estimation of Structure of Distribut- ed Lag}

Consider at first a case, when $X_{n}=X\left(u_{i, n}\right)$. Apply the approach, offered in [7, 29]. Generate set for an estimation of structure (1). Introduce a variable $s_{n}=I^{T} U_{n}$,

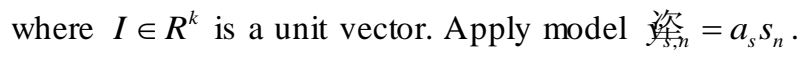
Find parameter $\hat{a}_{s} \in R$ from a condition

$$
\arg \min _{\hat{a}_{s}}\left(\hat{y}_{s, n}-y_{n}\right)^{2}=\hat{a}_{s}^{*} .
$$

Let $e_{n}=\hat{y}_{s, n}-y_{n}$ is a variable which contains the data about structure of a lag of system (1). As argument $X\left(u_{i, n}\right)$ use variable $u_{i, n} \in U_{n}$, which ensures maximum value of coefficient of determination $r_{e, u_{i}}^{2}$ between $e$ and $u_{i}$. It is true and for $y_{n}$. As shown in [7, 29], set $\left\{e_{n}, u_{i, n}\right\}$ does not allow to solve a problem of structural identification. The given statement follows directly from this that the index in an inequality of Holder [32] for $e_{n}$ is close to zero. Therefore introduce coefficient of structural properties (CSP) [7]

$$
k_{e, u_{i}, n}=k_{s}\left(e, u_{i}, n\right)=\frac{e_{n}}{u_{i, n}} .
$$

Generate set

$$
\mathrm{I}_{k}=\mathrm{I}_{k}(k, e)=\left\{e_{n}, k_{n}, n \in J_{N}=[0, N]\right\}, k_{n} \stackrel{\mathrm{df}}{=} k_{e, u_{i}, n} .
$$

Definition 2. Name $\mathscr{P}_{s}=(k, e)$ structural space of the system(1), allowing to identify structure of vector $X_{n}$.

On $J_{N}$ will order $k_{e, u_{i}, n}$ on increase. Generate $\left\{k_{q}^{v}\right\}$, where $k_{q}^{v}=k_{s}\left(e, u_{i}, q\right), q \in J_{N}^{v}=[0, N]$. As to every $k_{q}^{v}$ corresponds value $e_{q}^{v}$ receive

$$
\mathrm{I}_{k}^{v}=\left\{e_{q}^{v}, k_{q}^{v}, q \in J_{N}^{v}\right\} .
$$

In $\mathscr{P}_{s}$ define map $\Gamma_{e, k}^{v}:\left\{k_{q}^{v}\right\} \rightarrow\left\{e_{q}^{v}\right\}$ and structure $S_{k, e}^{v}$ corresponding to it.

Now estimate structure $X_{n}$ on the basis of the analysis $\mathrm{I}_{k}^{v}$ and $S_{k, e}^{v}$.

Such approach well works at an estimation of structure nonlinear static systems [7, 29]. For systems with the distributed lag it demands modification.

\section{Decision-Making on Length of a Lag in $X\left(u_{i, n}\right)$}

Set $\mathrm{I}_{e, u}=\left\{e_{n}, u_{i, n}\right\}$ contains uncertainty $\mathcal{N}_{L}$ and $\mathcal{N}_{\mathcal{L} g}$. $\mathcal{N}_{L}$ is an incomplete account of linear component system (1). $\mathcal{N}_{\mathcal{L g}}$ reflects influence of disturbance from the distributed lag. For an exception $\mathcal{N}_{\mathcal{L}}$ [28] construct a secant for $e_{n}$

$$
\bar{\gamma}\left(e, u_{i}\right)=\alpha_{0}+\alpha_{1} u_{i, n}
$$

where $\alpha_{0}, \alpha_{1}$ define as a solution of a problem (5).

Introduce new variable $\varepsilon_{n}=e_{n}-\bar{\gamma}\left(e, u_{i}\right)$ which does not contain $\mathcal{N}_{L}$. For estimation $\mathcal{N}_{\mathcal{L g}}$ analyze set $\left\{\varepsilon_{n}, u_{i, n}\right\}$.

To reception of a tentative estimation of the maximum lag $m$ apply algorithm $C D_{\rho}$ from [28].

In work the set-functional approach to structural identification is applied. Therefore known methods of a choice length the lag, based on statistical criteria (section 1 see), we do not consider.

To an estimation of independence of elements of a vector $X_{n}$ apply the theorem 1 . As the set analysis $\mathrm{I}_{e, u}$ 
is inefficient to eat, apply results of section 4 and generate set

$$
\mathrm{I}_{\varepsilon, k_{X}}=\left\{\varepsilon_{n}, k_{\varepsilon, x_{i, n}}, i=\overline{1, m} \quad \forall n \in J_{N}\right\},
$$

where $k_{\varepsilon, x_{i}, n}$ calculate on the basis of (6) and designate $e_{n}=\varepsilon_{n}, x_{i, n} \in X_{n}$.

On $\mathrm{I}_{\varepsilon, k_{X}}$ introduce transformation

$$
\Gamma_{\varepsilon, k_{\varepsilon, x_{i}}}:\left\{k_{\varepsilon, x_{i, n}}\right\} \rightarrow\left\{\varepsilon_{n}\right\}
$$

$\Gamma_{\varepsilon, k_{\varepsilon, x_{i}}}$ in space $\mathcal{P}_{s, \varepsilon}=\left(k_{\varepsilon, x_{i, n}}, \varepsilon\right)$ corresponds structure $S_{k_{\varepsilon, x_{i}}, \varepsilon}$. Construct secants for $S_{k_{\varepsilon, x_{i}}, \varepsilon}$

$$
\bar{\gamma}\left(\varepsilon_{n}, k_{\varepsilon, x_{i, n}}\right)=\beta_{i} k_{\varepsilon, x_{i, n}} \quad \forall i=\overline{1, m_{*}},
$$

where $\beta_{i}$ are defined as the solution of a problem (5); $m_{*}$ is a value $m$ received by means of algorithm $C D_{\rho}$.

Generate a vector

$$
\Phi_{\varepsilon, n}=\left[\begin{array}{c}
\bar{\gamma}\left(\varepsilon_{n}, k_{\varepsilon, x_{1, n}}\right), \bar{\gamma}\left(\varepsilon_{n}, k_{\varepsilon, x_{2, n}}\right), \\
\ldots, \bar{\gamma}\left(\varepsilon_{n}, k_{\varepsilon, x_{m, n}}\right)
\end{array}\right]^{T}
$$

and apply model $\hat{\varepsilon}_{n}=\Psi_{\varepsilon}^{T} \Phi_{\varepsilon, n}$ to change forecasting $\varepsilon_{n}$, where a vector $\Psi_{\varepsilon} \in R^{m_{*}}$ define on the basis of results section 3 .

Theorem 2 [28]. Let on set the $\mathrm{I}_{\varepsilon, k_{X}}$ field of secants is constructed for $\varepsilon_{n}$

$$
S_{\varepsilon}=\left\{\bar{\gamma}\left(\varepsilon_{n}, k_{\varepsilon, x_{i, n}}\right)\left(\forall i=\overline{1, m_{*}}\right) \&\left(n \in J_{N}\right)\right\}
$$

and the model $\hat{\varepsilon}_{n}=\Psi_{\varepsilon}^{T} \Phi_{\varepsilon, n}$ is applied to forecasting of change a variable $\varepsilon_{n}$. Then the vector $X_{n} \in R^{m_{*}}$ is an element of structure of system(1), if

$$
\sum_{i=1}^{m_{*}} \psi_{\varepsilon, i} \neq 1
$$

where $m_{*}$ is defined by means of algorithm, $C D_{\rho}$, $\psi_{\varepsilon, i} \in \Psi_{\varepsilon}$.

Remark 1. As $\varepsilon_{n}$ contains the information on vector influence $X_{n}$ at statistical treatment $U_{n}, \xi_{n}$ use analogue of criterion Durbin-Wats on criterion [28]

$$
d=\frac{\sum_{n=1}^{N} \varepsilon_{n}^{2}}{\sum_{n=1}^{N} e_{n}^{2}}
$$

Level of nonlinearity of system(1) in the presence of the distributed lag on $X_{n}$ define as

$$
\Delta\left(\Psi_{\varepsilon}\right)=\sum_{i=1}^{m_{*}} \psi_{\varepsilon, i}-1
$$

Theorem 3. Consider the set of secants $S_{\varepsilon}$ set on $\mathrm{I}_{\varepsilon, k_{x}}$, and a secant $\bar{\gamma}\left(e, k_{e, u_{i}}\right)=\alpha_{1} k_{e, u_{i}}$ for $S_{k_{e, u_{i}}, e}$. Let for secants coefficients of determinations are known $r_{\varepsilon, k_{x_{j}}}^{2}$, $j=\overline{1, m}$ and $r_{e, k_{u i}}^{2}$. Then the vector $X\left(u_{i, n}\right) \in R^{m}$ is an element of structure system(1), if $\forall z=\overline{1, m}$

$$
\left|r_{\varepsilon, k_{x_{z}}}^{2}-r_{e, k_{u i}}^{2}\right| \leq \delta_{x}
$$

where $\delta_{x} \geq 0$ is a set value.

At the structure analysis the distributed lag of system (1) interpret as a nonlinear component (1). It follows from the theorem 1. Therefore develop the procedure, allowing making the decision on an uncertainty class $\mathcal{N}_{\text {Lg }}$. More low the method of an estimation the distributed lag, based on development of the approach from [28] is offered.

Between $u_{i, n}$ and $u_{i, n-j}(j \geq 1)$ is a dependence and disturbance works $\xi_{n}$. Therefore application of secants a linear class $\bar{\gamma}$ can appear inefficient for decisionmaking. Consider set

$$
\mathrm{I}_{e, k_{X}}=\left\{e_{n}, k_{e, x_{\rho, n}}, \rho=\overline{1, m} \forall n \in J_{N}\right\},
$$

where $x_{\rho, n} \in X\left(u_{i, n}\right)$.

For every $\rho=\overline{1, m}$ pair $\left(e_{n}, k_{e, x_{\rho, n}}\right)$ will order on increase on $k_{e, x_{\rho, n}}$ and receive set

$$
\mathrm{I}_{e, k_{x_{\rho}}}^{v}=\left\{e_{\rho, q}^{v}, k_{e, x_{\rho, q}}^{v}, q \in J_{N}^{v}\right\}
$$

where the index $\rho$ in $e_{\rho, q}^{v}$ is introduced to underline dependence $e_{q}^{v}$ from $k_{e, x_{\rho, q}}^{v}$.

Corresponding set $\mathrm{I}_{k}^{v}$ construct for $u_{i}$. Consider mappings

$$
\Gamma_{e, e_{\rho}}^{v}:\left\{e_{q}^{v}\right\} \rightarrow\left\{e_{\rho, q}^{v}\right\}, \rho \geq 1
$$


Which describe structures $S_{e, e_{\rho}}^{v}$. For every $S_{e, e_{\rho}}^{v}$ construct a secant

$$
\bar{\gamma}_{2, \rho}\left(e_{q}^{v}, e_{\rho, q}^{v}\right)=a_{\rho, 0}^{v} e_{q}^{v}+a_{\rho, 1}^{v}\left(e_{q}^{v}\right)^{2}
$$

and define a coefficient of determination $r_{2, e^{v}, e_{\rho}^{v}}^{2}$.

Theorem 4. Let for system (1) with $X\left(u_{i, n} \in U_{n}\right) \in R^{m}$ : i) on sets $\mathrm{I}_{e, k_{x_{\rho}}}^{v}$ and $\mathrm{I}_{k}^{v}$ structures $S_{e, e_{\rho}}^{v}$, described by mappings (11), are constructed; ii) for $S_{e, e_{\rho}}^{v}$ secants $\bar{\gamma}_{2, \rho}\left(e_{q}^{v}, e_{\rho, q}^{v}\right)$ (12) are defined. If for a secant $\bar{\gamma}_{2, \rho}\left(e_{q}^{v}, e_{\rho, q}^{v}\right)$ structure the $S_{e, e_{\rho}}^{v}(\rho \geq 1)$ condition is executed

$$
r_{2, e^{v}, e_{\rho}^{v}}^{2} \geq \delta_{v}
$$

where $\delta_{v}>0$ is some set number, then is $x_{\rho, n}=u_{i, n-\rho}$ element of structure system(1).

The theorem 4 allows to define length of the distributed lag $m_{*}$ of a variable $u_{i} \in U$. The decision accept on the basis of the interdependence analysis between a virtual variable $e_{n}$ and its forecast by means of variables $x_{\rho, n}$.

The decision on lag presence on $u_{i}$ can be received on the basis of the analysis of the data in space $\mathscr{P}_{s}=(k, e)$.

Consider set $\mathrm{I}_{k_{i}}^{v}=\left\{e_{q}^{v}, k_{i, q}^{v}, q \in J_{N}^{v}\right\}$ and define on it mapping $\Gamma_{e, k_{i}}^{v}:\left\{k_{i, q}^{v}\right\} \rightarrow\left\{e_{q}^{v}\right\}$, where $k_{i, q}^{v}$ is a coefficient of structural properties on an input $u_{i}$. On a basis $\Gamma_{e, k_{i}}^{v}$ set structure $S_{e, k_{i}}^{v}$. Generate sets $\mathrm{I}_{e, k_{x_{\rho}}}^{v}$ and on them define $\Gamma_{e, k_{x_{\rho}}}^{v}$ and $S_{e, k_{e, x_{\rho}}}^{v}, \rho \geq 1$. Define for $S_{e, k_{i}}^{v}$ and $S_{e, k_{e, x_{\rho}}}^{v}$ secants

$$
\begin{aligned}
& \bar{\gamma}_{2}\left(e_{i, q}^{v}, k_{i, q}^{v}\right)=\bar{\gamma}_{2, i}=a_{i, 0}^{v} k_{i, q}^{v}+a_{i, 1}^{v}\left(k_{i, q}^{v}\right)^{2}, \\
& \bar{\gamma}_{2}\left(e_{x_{\rho}, q}^{v}, k_{x_{\rho}, q}^{v}\right)=\bar{\gamma}_{2, x_{\rho}}=\tilde{a}_{\rho, 0}^{v} k_{x_{\rho}, q}^{v}+\tilde{a}_{\rho, 1}^{v}\left(k_{x_{\rho}, q}^{v}\right)^{2},
\end{aligned}
$$

where $a_{i, 0}^{v}, a_{i, 1}^{v}, \tilde{a}_{\rho, 0}^{v}, \tilde{a}_{\rho, 1}^{v}$ are some numbers defined by means of least-squares method. For $\bar{\gamma}_{2}\left(e_{i, q}^{v}, k_{i, q}^{v}\right)$ and $\bar{\gamma}_{2}\left(e_{x_{\rho}, q}^{v}, k_{x_{\rho}, q}^{v}\right)$ coefficients of determinations also are known $r_{2, \bar{\gamma}_{2, i}, e^{v}}^{2}, r_{2, \bar{\gamma}_{2, \rho}, e^{v}}^{2}$. Designate average values $x_{\rho, n}$ as $\bar{x}_{\rho, n}$.
Theorem 5. Let for system (1) with $X\left(u_{i, n} \in U_{n}\right) \in R^{m}$ in space $\left.\mathscr{P}_{s}=(k, e): \mathrm{i}\right)$ on sets $\mathrm{I}_{e, k_{x_{\rho}}}^{v}, \mathrm{I}_{k_{i}}^{v}$ structures $S_{e, k_{i}}^{v}$ and $S_{e, k_{i}}^{v}$, described by mappings $\Gamma_{e, k_{x_{\rho}}}^{v}$ and $\Gamma_{e, k_{i}}^{v}$, are constructed; ii) for $S_{e, k_{i}}^{v}$ and $S_{e, k_{e, x_{\rho}}}^{v}$ secants (13), (14) are defined. If for a secant $\bar{\gamma}_{2}\left(e_{x_{\rho}, q}^{v}, k_{x_{\rho}, q}^{v}\right)$ structure the $S_{e, k_{e, x_{\rho}}}^{v}(\rho \geq 1)$ condition are executed

$$
\begin{aligned}
& \left|r_{2, \bar{\gamma}_{2, x_{\rho}}, e_{1}^{v}}^{2}-r_{2, \bar{\gamma}_{2, i}, e_{1}^{v}}^{2}\right| \leq \delta_{\rho}, \\
& \left|\tilde{a}_{\rho, 1}^{v}-\bar{x}_{\rho, n}\right| \leq \pi_{\rho},
\end{aligned}
$$

where $\delta_{\rho}>0$ and $\pi_{\rho}$ are some set numbers, then is $x_{\rho, n}=u_{i, n-\rho}$ element of structure of system(1).

The proof of the theorem 5 is obvious. It is based that structures $S_{e, k_{i}}^{v}$ and $S_{e, k_{e, x_{\rho}}}^{v}$ describe variable change $e_{q}^{v}$. Therefore the decision on inclusion in structure of system (1) accept concerning that variable $x_{\rho} \in X_{n}$, which ensures necessary level of a coefficient determination. Values $r_{2, \bar{\gamma}_{2, \rho}, e^{v}}^{2}$ should belong to the set interval, as reflects (15). If values $r_{2, \bar{\gamma}_{2, \rho}, e^{v}}^{2}$ are approximate, then use restriction (16) on a received estimation of average value $x_{\rho, n}=u_{i, n-\rho}$.

Other approaches of a choice of leng th the distributed lag $u_{i}$ are described in [28].

\section{Choice of Length Lag for $X\left(y_{n}\right)$}

Let in system (1) $X_{n}=\left[x_{1, n}, x_{1, n}, \ldots, x_{l, n}\right]^{T}$, where $x_{i, n}=y_{n-i}, i=\overline{1, l}$. To a prior estimation $l$ apply algorith m $C D_{\rho}$. One of methods of a choice of length the distributed lag is described in [28]. It is based on application of the theorem 1 . As show results of modelling, to make the decision on structure of the distributed lag on $y_{n}$ in space $\mathscr{P}_{s}=(k, e)$ on a class of linear secants for virtual portraits $S_{e, k}^{v}$ not always it is possible. Therefore we describe the approach based on application of theorems 4,5 .

Consider space $\mathscr{P}_{s, e}=\left(e_{q}^{v}, e_{i, q}^{v}\right)$, where $e_{q}^{v}, e_{i, q}^{v}$ are elements of sets

$$
\begin{gathered}
\mathrm{I}_{e, k_{y}}^{v}=\left\{e_{q}^{v}, k_{e, y_{q}}^{v}, q \in J_{N}^{v}\right\}, \\
\mathrm{I}_{e, k_{x_{i}}}^{v}=\left\{e_{i, q}^{v}, k_{e, x_{i, q}}^{v}, q \in J_{N}^{v}\right\}, i \geq 1 .
\end{gathered}
$$


On $\mathrm{I}_{e, k_{y}}^{v}, \mathrm{I}_{e, k_{x_{i}}}^{v}$ introduce mapping $\Gamma_{e, e_{i}}^{v}:\left\{e_{q}^{v}\right\} \rightarrow\left\{e_{i, q}^{v}\right\}$, $i \geq 1$. The method of its construction is described in section 5. $\Gamma_{e, e_{i}}^{v}$ corresponds structure $S_{e, e_{i}}^{v}$. For every $S_{e, e_{i}}^{v}$ construct a secant

$$
\bar{\gamma}_{2, i}\left(e_{q}^{v}, e_{i, q}^{v}\right)=a_{i, 0}^{v} e_{q}^{v}+a_{i, 1}^{v}\left(e_{q}^{v}\right)^{2}
$$

and define a coefficient of determination $r_{2, e^{v}, e_{i}^{v}}^{2}$. Apply the theorem 4 to an estimation of length the distributed lag on $y_{n}$.

Consider space $\mathscr{P}_{s, k_{x_{i}}, e}=\left(k_{e, x_{i}}^{v}, e_{i}^{v}\right)$. On set $\mathrm{I}_{k_{x_{i}}}^{v}$ define mapping $\Gamma_{e, k_{x_{i}}}^{v}:\left\{k_{x_{i}, q}^{v}\right\} \rightarrow\left\{e_{q}^{v}\right\}$ and a virtual portrait $S_{e, k_{e, x_{i}}}^{v}$, where $k_{x_{i}, q}^{v}$ is a coefficient of structural properties on an input $x_{i}$. Also consider set

$$
\mathrm{I}_{e, k_{y}}^{v}=\left\{e_{q}^{v}, k_{e, y, q}^{v}, q \in J_{N}^{v}\right\}
$$

introduce mapping $\Gamma_{e, k_{e, y}}^{v}:\left\{k_{e, y, q}^{v}\right\} \rightarrow\left\{e_{q}^{v}\right\}$ and structure $S_{e, k_{e, y}}^{v}$. Apply (13), (14) and define secants

$$
\begin{aligned}
& \bar{\gamma}_{2}\left(e_{q}^{v}, k_{e, y, q}^{v}\right)=\bar{\gamma}_{2}=a_{y, 0}^{v} k_{e, y, q}^{v}+a_{y, 1}^{v}\left(k_{e, y, q}^{v}\right)^{2}, \\
& \bar{\gamma}_{2}\left(e_{i, q}^{v}, k_{x_{i}, q}^{v}\right)=\bar{\gamma}_{2, i}=\tilde{a}_{i, 0}^{v} k_{x_{i}, q}^{v}+\tilde{a}_{i, 1}^{v}\left(k_{x_{i}, q}^{v}\right)^{2},
\end{aligned}
$$

where $a_{y, 0}^{v}, a_{y, 1}^{v}, \tilde{a}_{i, 0}^{v}, \tilde{a}_{i, 1}^{v}$ are some numbers defined by means of a method least-squares method.

For $\bar{\gamma}_{2}\left(e_{q}^{v}, k_{e, y, q}^{v}\right)$ and $\bar{\gamma}_{2}\left(e_{i, q}^{v}, k_{x_{i}, q}^{v}\right)$ coefficients of determinations also are known $r_{2, \bar{\gamma}_{2}, e^{v}}^{2}, r_{2, \bar{\gamma}_{2, i}, e^{v}}^{2}$. Designate average values $x_{i, n}$ as $\bar{x}_{i, n}$. Apply the theorem 5 and receive an estimation of length the distributed lag on $y_{n}$.

The offered approach apply in a case

$$
X_{n}=X\left(u_{i, n} \in U_{n}, y_{n}\right) \in R^{m}
$$

It is possible to apply Lyapunov characteristic indicators to an estimation of the distributed lag [33]. For this purpose use the approach described in [28, 29].

\section{About Sector Sets}

On the basis of the analysis of sector sets (SS) [29, 30] we can make the decision on a nonlinearity class. Despite complexities their construction for irregular inputs
SS allow to solve problems of structural identification of nonlinear static systems. Attempts to extend this approach on systems with the distributed lag have appeared unsuccessful. Explain it to that the distributed lag is result of the decision of difference equations. The received decision is somewhat approximate to a variable which is a basis for lag reception. Therefore SS in structural space for a base variable and its lags will differ not strongly. It does not allow developing effective algorith ms of decision-making. Presence of the distributed lags on variables $y_{n}$, is the reason of decrease a coefficient of determination between input variables and a systemoutput.

\section{About Estimations of Parameters of System (1) at Distributed Lags}

Identification of parameters in system with the distributed lags will execute on a class of a priori set models of parameters (look section 1). Such approach it is based on minimization of number of the estimated parameters in the conditions of a mult icollinearity. It is the dominating. Authors offer various variations of approaches within the limits of the given concept of identification [17]. In [28] the method of identification the distributed lag on the basis of application Lyapunov characteristic indicators is described. It is based on estimations of a coefficient structural properties system on the set input. The coefficient structural properties is a parameter estimation at the considered distributed lag. Here we give development of the given approach.

We accept the received estimations of an average coefficient structural properties for admis sible estimations of parameters at the distributed lags and form a vector $\breve{B} \in R^{m}$. Introduce some limited vector $\Theta \in R^{h}$, where $h<m, \Theta \in \Omega_{\Theta}$. In particular, at $h=1$,

$$
\theta \in \Omega_{\Theta}=\left\{\theta \in R, \theta \in\left[1-\varepsilon_{h}, 1+\varepsilon_{h}\right]\right\},
$$

$\varepsilon_{h} \geq 0$ is some set number. The second composed in the right part (1) write down in a form

$$
B^{T} X_{n}=\theta \breve{B}^{T} X_{n}
$$

Apply a least-squares method (LSM) and receive an estimation $\theta$.

If adequacy of model with an estimation $\theta$ is unsatisfactory, then choose $h>1$. Consider a vector $\Theta$ and execute division $X_{n}$ into parts so that they contained nonsingular components. Let, for example $X_{n}=\left[X_{1, n}^{T}, X_{2, n}^{T}\right]^{T}$. Then $\breve{B}=\left[\breve{B}_{1}^{T}, \breve{B}_{2}^{T}\right]^{T}$. Let

$$
\Theta=\left[\theta_{1}, \theta_{2}\right]^{T}, D(\Theta)=\theta_{1} I_{h_{1}} \dot{+} \theta_{2} I_{h_{2}},
$$


where $h_{1}+h_{2}=h, D(\Theta)$ is a diagonal matrix from a vector $\Theta, I_{h_{1}} \in R^{h_{1} \times h_{1}}$ is an unity matrix, $\dot{+}$ is a direct sum of matrixes.

Then instead of (20) in model of identification for $B^{T} X_{n}$ receive a relation

$$
B^{T} X_{n}=\left[\theta_{1} \breve{B}_{1}^{T}, \theta_{2} \breve{B}_{2}^{T}\right]\left[X_{1, n}^{T}, X_{2, n}^{T}\right]^{T} .
$$

To a vector estimation $\Theta$ apply LSM. Estimate adequacy of the received model. If the forecasting error appears is big, then change dimension of vector $\Theta$. Execute identification process again.

Remark 2. A vector $\Theta$ is possible to identify by means of adaptive algorithm taking into account restriction $\Theta \in \Omega_{\Theta}$. To synthesis of procedure identification apply a method $\varphi$-algorithms [34].

\section{Examples}

Consider system (1) with $A=[1.5 ; 2 ; 2.8]^{T}$, $B=\left[0.7 ; 0.6^{\top}\right], X_{n} \in R^{2}, X_{n}=X\left(u_{1, n}\right) . u_{i, n} \in U_{n} \in R^{3}$ are limited stochastic functions, $\xi_{n}$ is a random variable with a zero expectancy and a final dispersion, $\left|\xi_{n}\right| \leq 0.3$. $u_{1, n}$ receive on a basis the decision difference the equations of the second order. Apply the theorem 1 and receive, that the system is nonlinear, $\Delta(\Psi)=0.03$. Value $\Delta(\Psi)$ explain presence of linear interdependences (multicollinearity) in system. For lag estimation generate set

$$
\mathrm{I}_{e, u}=\left\{e_{n}, u_{i, n}(\forall i=\overline{1,3}) \&\left(\forall n \in J_{N}\right)\right\}
$$

The analysis $\mathrm{I}_{e, u}$ has shown, that the lag is at a variable $u_{1, n}$. Apply algorithm $C D_{\rho}$ and receive a prior estimation of length of a lag $m$. The estimation of length of a lag on $u_{1, n}$ is $m^{*}=2$. To validation of the received result apply the theorem 4 .

Generate sets $\mathrm{I}_{e, k_{x_{\rho}}}^{v}, \mathrm{I}_{k}^{v}$, consider mappings (11) and structures $S_{e, e_{\rho}}^{v}$, where $\rho \geq 1$. For $S_{e, e_{\rho}}^{v}$ define secants $\bar{\gamma}_{2, \rho}\left(e_{q}^{v}, e_{\rho, q}^{v}\right)$ and $r_{2, e^{v}, e_{\rho}^{v}}^{2}$ :

$$
\begin{aligned}
& \bar{\gamma}_{2,1}\left(e_{q}^{v}, e_{1, q}^{v}\right)=0.975 e_{q}^{v}-0.0133\left(e_{q}^{v}\right)^{2}, r_{2, e^{v}, e_{1}^{v}}^{2}=0.948, \\
& \bar{\gamma}_{2,2}\left(e_{q}^{v}, e_{2, q}^{v}\right)=0.967 e_{q}^{v}-0.019\left(e_{q}^{v}\right)^{2}, r_{2, e^{v}, e_{2}^{v}}^{2}=0.934,
\end{aligned}
$$

$$
\bar{\gamma}_{2,3}\left(e_{q}^{v}, e_{3, q}^{v}\right)=0.954 e_{q}^{v}-0.0038\left(e_{q}^{v}\right)^{2}, r_{2, e^{v}, e_{3}^{v}}^{2}=0.91
$$

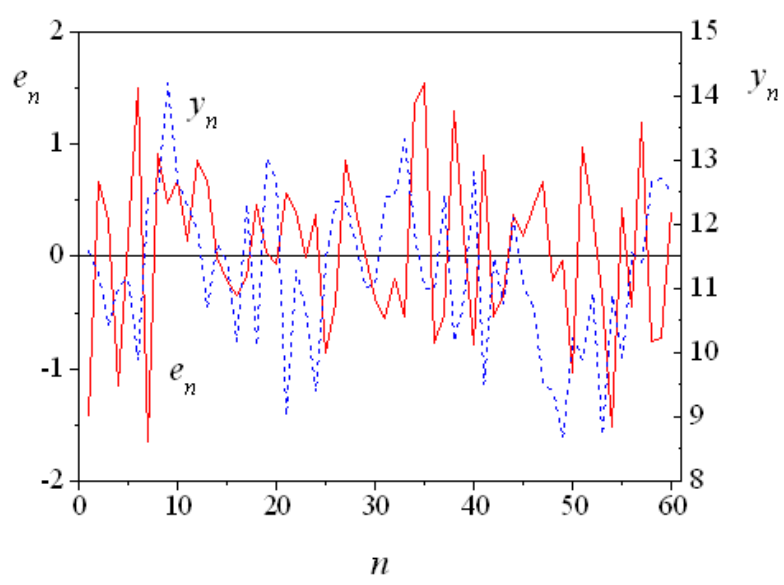

Fig. 1: Change $y_{n}, \varepsilon_{n}$

Let $\delta_{v}=0.93$. Apply the theorem 4 and receive $m^{*}=2$. On Figure 1 show lag influence on $u_{1, n}$.

To check of the received estimation of a lag apply theorems 5. Define secants (13), (14) and coefficients of determinations

$$
\begin{aligned}
& \bar{\gamma}_{2}\left(e_{1, q}^{v}, k_{1, q}^{v}\right)=\bar{\gamma}_{2,1}=2.13 k_{i, q}^{v}-0.793\left(k_{1, q}^{v}\right)^{2}, \\
& r_{2, \bar{\gamma}_{2,1}, e_{1}^{v}}^{2}=0.971, \\
& \bar{\gamma}_{2}\left(e_{x_{1}, q}^{v}, k_{x_{1}, q}^{v}\right)=\bar{\gamma}_{2, x_{1}}=1.93 k_{x_{1}, q}^{v}+0.48\left(k_{x_{1}, q}^{v}\right)^{2}, \\
& r_{2, \bar{\gamma}_{2, x_{1}}, e_{x_{1}}^{v}}^{2}=0.964, \\
& \bar{\gamma}_{2}\left(e_{x_{2}, q}^{v}, k_{x_{2}, q}^{v}\right)=\bar{\gamma}_{2, x_{2}}=1.76 k_{x_{2}, q}^{v}+0.22\left(k_{x_{2}, q}^{v}\right)^{2}, \\
& r_{2, \bar{\gamma}_{2, x_{2}}, e_{x_{2}}^{v}}^{2}=0.928, \\
& \bar{\gamma}_{2}\left(e_{x_{3}, q}^{v}, k_{x_{3}, q}^{v}\right)=\bar{\gamma}_{2, x_{3}}=1.69 k_{x_{3}, q}^{v}+0.053\left(k_{x_{3}, q}^{v}\right)^{2}, \\
& r_{2, \bar{\gamma}_{2, x_{3}}, e_{x_{3}}^{v}}^{2}=0.922 .
\end{aligned}
$$

Average values for $u_{1}$ and $x_{\rho}: \bar{u}_{1}=2.026$, $\bar{x}_{1}=2.011, \bar{x}_{2}=1.996, \bar{x}_{3}=1.98$. Let $\delta_{\rho}=0.043$, $\pi_{\rho}=0.02$. Check up conditions (15), (16). They will be executed for lags $x_{1}, x_{2}$. So, we have received estimations for length of the distributed lag on an input $u_{1}$.

On Figure 2 show results of application the theorem 5. They confirm a conclusion about influence of the distributed lag as some equivalent nonlinearity in system. This conclusion receives from the analysis of change 
secant $\bar{\gamma}_{2,1}$ of structure $S_{e, k_{1}}^{v}$. For $\bar{\gamma}_{2, x_{3}}$ we have almost linear dependence that confirms an elimination of a lag $x_{3}$ of model structure.

Consider system (1) with $X_{n}=\left[x_{1, n}, x_{2, n}\right]^{T}$, $x_{1, n}=y_{n-1}, x_{2, n}=u_{1, n-1}$. Execute the analysis of set (21) and apply algorithm $C D_{\rho}$ to an estimation of the dis- tributed lag. Results of the analysis give lags on $y_{n}$ and $u_{1, n}$. The estimation is $m^{*}=1$ on these variables. Apply the theorem 4 and confirm this conclusion.

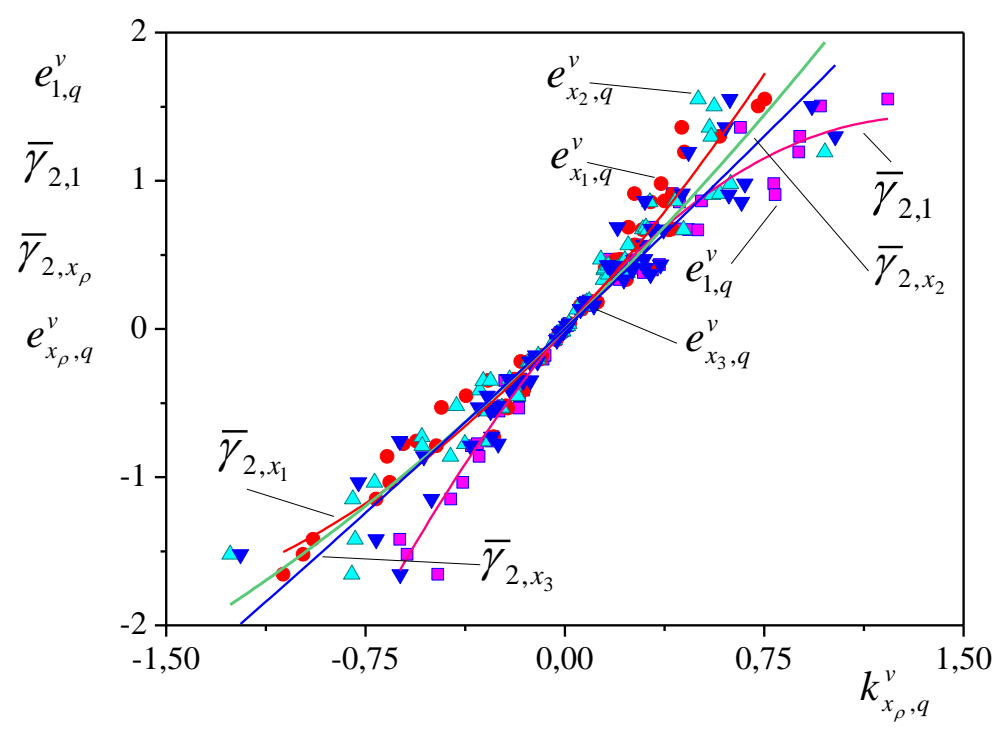

Fig. 2: Structures $S_{e, k_{i}}^{v}$ and secants $\bar{\gamma}_{2, x_{\rho}}$

Define secants (13), (14) and coefficients of determinations

$$
\begin{aligned}
& \bar{\gamma}_{2}\left(e_{1, q}^{v}, k_{1, q}^{v}\right)=\bar{\gamma}_{2,1}=1.909 k_{i, q}^{v}-0.567\left(k_{1, q}^{v}\right)^{2}, \\
& r_{2, \bar{\gamma}_{2,1}, e_{1}^{v}}^{2}=0.933, \\
& \bar{\gamma}_{2}\left(e_{x_{2}, q}^{v}, k_{x_{2}, q}^{v}\right)=\bar{\gamma}_{2, x_{2}}=2.0 k_{x_{2}, q}^{v}+0.771\left(k_{x_{2}, q}^{v}\right)^{2}, \\
& r_{2, \bar{\gamma}_{2, x_{2}}, e_{x_{2}}^{v}}^{2}=0.965, \\
& \bar{\gamma}_{2}\left(e_{x_{2,2}, q}^{v}, k_{x_{2}, q}^{v}\right)=\bar{\gamma}_{2, x_{2,2}}=1.694 k_{x_{2,2}, q}^{v}+0.115\left(k_{x_{2,2}, q}^{v}\right)^{2}, \\
& r_{2, \bar{\gamma}_{2, x_{2},}, e_{x_{2,2}}^{v}}^{2}=0.946,
\end{aligned}
$$

where $x_{2,2, q}=u_{1, q-2}$.

Average values for $u_{1, n}, x_{2, n}$ and $x_{2,2, n}: \bar{u}_{1}=2.028$, $\bar{x}_{2}=2.023, \bar{x}_{2,2}=2.0$. Secants $\bar{\gamma}_{2,1}, \bar{\gamma}_{2, x_{2}}$ corroborate lag presence on $u_{1, n}$. The secant $\bar{\gamma}_{2, x_{2,2}}$ is almost linear (for a linear secant $\bar{\gamma}_{1, x_{2,2}} r_{2, \bar{\gamma}_{1, x_{2,2}}, e_{x_{2,2}}^{v}}^{2}=0.944$ ). Condition
(16) for $\bar{\gamma}_{2, x_{2,2}}$ with $\pi_{\rho}=0.15$ is not true. Therefore $x_{2,2, n}$ is no element of structure of system (1). High $r_{2, \bar{\gamma}_{1, x_{2,2}}, e_{x, 2}^{v}}^{v}$ explain by means of application of the statement from [28]. Similarly execute identification of the distributed lag on $y_{n}$.

So, results of modelling confirm efficiency of the offered algorithms and methods.

\section{Conclusion}

In work is functional-set approach to structural identification of discrete systems with the distributed lag is offered. The decisions on structure lag system part accept on the basis of the analysis special static structures. It is shown that the distributed lag can be considered as nonlinearity. We have applied to decision-making on length of a lag secants of the second order and have made the analysis of change their parameters. It is shown, that as criterion of decision-making on a belonging of the distributed lag the system structure can use degree of linearity of a secant the second order. The approach to parametrical identification of factors model is offered at the distributed lags. Unlike existing approaches we do not do any assumptions concerning the 
law of change parameters. The approach is based on definition of tentative estimations parameters model on the basis of the analysis of a coefficient structural properties system. These estimations are specified on the basis of identification auxiliary factors (multiplicate parameters). Results of modelling have confirmed working capacity of the offered methods and alg orithms

\section{References}

[1] Malinvaud, E. Statistical methods in econometrics. 3d ed. North-Holland Publishing Co, Amsterdam, 1980.

[2] Johnston J. Econometric methods. 2nd edition. McGraw-Hill Book Company, New York, 1972.

[3] Demetriou I. C., Vassiliou E. E. An algorith m for distributed lag estimation subject to piecewise monotonic coefficients. International Journal of Applied Mathematics, v39, n1, 2009, pp. 1-10.

[4] Dhrymes, P.J. Distributed Lags: Problems of Estimation and Formulation. Holden-Day, San Francisco. 1971.

[5] Gershenfeld, N., The Nature of Mathematical Modelling. Cambridge University Press, Cambridge, 1999.

[6] Kailath, T. (editor), Linear Least-Squares Estimation, Stroudsburg, Pennsylvania: Dowden, Hutchinson and Ross, Inc., Benchmark Papers in Electrical Engineering and Computer Science, V17, 1977.

[7] Karabutov, N.N. Structural identification of systems: the analysis of informational structures, URSS, Librokom, Moscow, 2009. (in Russia).

[8] Armstrong, B. Models for the relationship between ambient temperature and daily morta lity. Epidemiology, v17(6), 2006, pp. 624-631.

[9] Nelson, C. R. Schwert, G.W. Estimating the parameters of a distributed lag model from cross section data: The case of hospital admissions and discharges. Journal of the American Statistical Association, v69, n347, 1974, pp. 627-633.

[10] Gasparrini A., Armstrong B., Kenwardb M. G. Distributed lag non-linear models. Statistics in Medicine, v29(21), 2010, pp. 2224-2234.

[11] Fisher, I. Note on a Short-cut Method for Calculating Distributed Lags. Bulletin de 1'Institut International de Statistique, v29, 1937.

[12] Коуск, L. M., Distributed Lags and Investment Analysis, North-Holland Publishing Company, Amsterdam, 1954.

[13] Almon, S. The distributed lag between capital appropriations and expenditures, Econo metrica, v33, 1965, pp. 178-196.
[14] Theil, H., Stern, R. M., A simple unimodal lag distribution. Metroeconomica, v 12, 1960, pp. 111119.

[15] Solow, R. On a family of lag distributions. Econometrica, v28, 1960, pp. 393-406.

[16] Jorgenson, D. W. Minimum variance, linear, unbiased seasonal adjustment of economic time series. Journal of the American Statistical Association, v59, n307, 1964, pp.681-724.

[17] Demetriou I. C., E. E. Vassiliou. A distributed lag estimator with piecewise monotonic coefficients. Proceedings of the world congress on engineering. 2008, v2, WCE 2008, July 2 - 4, 2008, London, U.K.

[18] Yoder J. Autoregressive distributed lag models. WSU Econometrics II, 2007, pp.91-115.

[19] Cheng Hsiao. Analysis of Panel Data. Cambridge University Press, 2003.

[20] Wen-Jen Tsay. The long memory autoregressive distributed lag model and its application on congressional approval. Institute of Economics, Academia Sinica, 2005.

[21] Carter R. A. L., Zellner A., The arar error model for univariate time series and distributed lag models.

http://faculty.chicagobooth.edu/arnold.zellner/mor e/CURRENT-PAPERS/ara rerrm.pdf

[22] Lansing K. J. Real-time estimation of trend output and the illusion of interest rate smoothing. FRBSF economic Review, 2002, pp. 18-34.

[23] Duffee Gr. R. Term structure estimation without using latent factors. Haas School of Business University of California-Berkeley, 2005.

[24] Campos J., Ericsson N. R., and Hendry D. F. General-to-specific modeling An overview and selected bibliography. International finance discussion papers, n838, 2005.

[25] Hansen B.E. Econometrics. University of Wisconsin, 2013.

[26] Alessi L., Barigozzi M. and Capasso M. A robust criterion for determining the number of static factors in approximate factor models. Working paper series, n903, 2008.

[27] Castle, J.L., Doornik, J.A., and Hendry, D.F. Evaluating automatic model selection. (Discussion paper series, 474) (workingPaper) Economists Online, 2010.

[28] Karabutov N.N. Structural identification of static systems with distributed lags. International journal of control science and engineering, v2012, n2(6), pp. 136-142. 
[29] Karabutov, N.N. Structural identification of static plants: Fields, structures, methods, URSS, Librokom, Moscow, 2011.

[30] Karabutov N.N. Structures, fields and methods of identification of nonlinear static systems in the conditions of uncertainty. Intelligent Control and Automation, v1, 2010, pp. 59-67.

[31] Karabutov N.N. Adaptive algorithms for structural parameters identification of single-valued nonlinearities of static systems with a vectorial input. Int. J. Sensing, Computing and Control, v2, n1, 2012, pp. $1-11$,

[32] Shilov, G. Mathematical analysis. Fizmathlit, Moscow 1961. (in Russia).

[33] Lyapunov, A.M., General problem about a movement stability. State publishing house of technikotheoretical literature, Moscow, 1950.

[34] Karabutov N.N. adaptive identification of systems. Information synthesis. URSS, Librokom, Moscow, 2006.

\section{Author's Profiles}

Nikolay Karabutov is the professor of department Problem of control of Moscow state institute radio engineering, electronics and automation. The professor chair of mathematics of Financial University under the Government of the Russian Federation. Dr.Sci.Tech. The research area is the automatic control theory, identification, adaptive control and decision-making.

How to cite this paper: Nikolay Karabutov,"Structural Identification of Systems with Distributed Lag", International Journal of Intelligent Systems and Applications(IJISA), vol.5, no.11, pp.1-10, 2013. DOI: 10.5815/ijisa.2013.11.01 